\title{
E-cigarettes helped more smokers quit than nicotine replacement therapy
}

\author{
Rob Cook clinical director ${ }^{1}$, Peter Davidson clinical adviser ${ }^{2}$, Rosie Martin clinical specialist ${ }^{1}$, on \\ behalf of NIHR Dissemination Centre
}

${ }^{1}$ Bazian, Economist Intelligence Unit healthcare, London, UK; ${ }^{2}$ Wessex Institute, University of Southampton, Southampton, UK

\begin{abstract}
The study

Hajek P, Phillips-Waller A, Przulj D, et al. A randomised trial of e-cigarettes versus nicotine-replacement therapy. N Engl J Med 2019;380:629-37.

This trial was funded by the NIHR Health Technology Assessment Programme (12/167/135) and by Cancer Research UK.

To read the full NIHR Signal, go to https://discover.dc.nihr.ac.uk/content/ signal-000757/e-cigarettes-helped-more-smokers-quit-than-nicotinereplacement-therapy
\end{abstract}

\section{Why was the study needed?}

In 2016, $15.5 \%$ of adults smoked tobacco. Although smoking is in decline, it remains the leading preventable cause of illness and premature death in the UK. Electronic cigarettes, used by around $6 \%$ of UK adults, are thought to be much less harmful than smoking tobacco. Controversy exists, however, over their effectiveness in attempts to quit, with different evidence reviews showing conflicting results.

There are few good quality trials, according to a recent evidence review. It identified 14 systematic reviews, seven of which included a meta-analysis. Of these, two found a positive effect, four found an inconclusive effect, and one found a negative effect.

This new study is the first sizeable, long term trial comparing modern refillable e-cigarettes with nicotine replacement therapy (NRT) within the context of existing NHS stop smoking services, where only about $10 \%$ of smokers manage to quit with standard care. The trial offers the best evidence yet available about the effectiveness of e-cigarettes combined with behavioural support for smoking cessation.

\section{What did this study do?}

In this randomised controlled trial, adult smokers (not pregnant or breastfeeding) were recruited through NHS stop smoking services in London, Leicester, and East Sussex. Researchers included only people with no strong preference as to whether they used e-cigarettes or NRT.

Participants were randomly assigned to NRT or e-cigarettes. NRT users were offered three months of their chosen product and were encouraged to choose a combination of quick acting and steady release products. E-cigarette users were given a refillable e-cigarette kit and bottle of nicotine liquid, with instructions to purchase additional liquid of their choice.

All participants were offered weekly one-to-one support for four weeks by a health professional and had carbon monoxide measured as a proxy for smoking during this period. They were followed up at 26 and 52 weeks. Of the 886 people randomised, $78.8 \%$ completed 12 months follow-up. Those who did not complete follow-up were assumed to have resumed smoking cigarettes. Although the drop-out rate was high, it is comparable with other trials of smoking cessation.

\section{What did it find?}

- Eighteen per cent of participants randomised to receive e-cigarettes reported themselves to be abstinent from smoking after 12 months, backed up by negative carbon monoxide breath tests. Of these people, $80 \%$ were still using e-cigarettes. In total, $39.5 \%$ of e-cigarette users were still using them after 12 months.

- Of participants randomised to receive NRT products, $9.9 \%$ reported themselves to be abstinent from smoking after 12 months, with negative carbon monoxide breath tests. Of these, $9 \%$ were still using NRT. In total, $4.3 \%$ of NRT users were still using products after 12 months.

- The chances of having stopped smoking were $83 \%$ higher for people who used e-cigarettes compared with NRT (relative risk $1.83,95 \%$ confidence interval 1.30 to 2.58 ).

- There were no serious adverse events related to either NRT use or e-cigarette use, although there were reports of respiratory events in both groups. NRT users were more likely to report 
nausea (37.9\% versus $31.3 \%$ of e-cigarette users). E-cigarette users were more likely to report mouth or throat irritation $(65.3 \%$ versus $51.2 \%$ of NRT users).

\section{What does current guidance say on this issue?}

The March 2018 evidence review from Public Health England says, "Stop smoking practitioners and health professionals should provide behavioural support to smokers who want to use an e-cigarette to help them quit smoking," and that they should receive training to provide support in the use of e-cigarettes as a stop smoking aid.

In March 2018 guidance, the National Institute for Health and Care Excellence recommends NRT or varenicline, with a referral to NHS stop smoking services, for smokers who wish to quit. Advice on e-cigarettes is to explain that "many people have found them helpful to quit smoking cigarettes" and "the evidence suggests that e-cigarettes are substantially less harmful to health than smoking but are not risk free."

\section{What are the implications?}

The finding that e-cigarettes are substantially more effective than NRT products, even when used with expert support, signals a potential shift towards provision of e-cigarettes as part of NHS stop smoking services.

This would require training of stop smoking service staff. Potential for purchase of e-cigarettes by services would need to be considered.
This publication did not include a cost comparison, but reports that the 2016 cost to the trial of an e-cigarette starter pack was $£ 30.25$, compared with $£ 120$ for a three month supply of a single NRT product. This suggests that e-cigarettes might be a good economic choice, as well as more clinically effective, compared with NRT.

Competing interests The $B M J$ has judged that there are no disqualifying financial ties to commercial companies. The authors declare the following other interests: none.

Further details of the BMJ policy on financial interests is here: https://www.bmj. com/about-bmj/resources-authors/forms-policies-and-checklists/declarationcompeting-interests

\section{Contributors Anna Sayburn}

All authors contributed to the development and review of this summary, as part of the wider NIHR Signals editorial team (https://www.bmj.com/NIHR-signals). RC is guarantor.

Disclaimer NIHR Signals are owned by the Department of Health and Social Care and are made available to the BMJ under licence. NIHR Signals report and comment on health and social care research but do not offer any endorsement of the research. The NIHR assumes no responsibility or liability arising from any error or omission or from the use of any information contained in NIHR Signals.

Permission to reuse these articles should be directed to disseminationcentre@nihr.ac.uk.

National Institute for Health and Care Excellence. Stop smoking interventions and services. NG92. London, 2018. https://www.nice.org.uk/guidance/ng92

2 McNeill A, Brose LS, Calder R, etal . Evidence review of e-cigarettes and heated tobacco products 2018. A report commissioned by Public Health England. Public Health England, 2018

Published by the BMJ Publishing Group Limited. For permission to use (where not already granted under a licence) please go to http://group.bmj.com/group/rights-licensing/ permissions 